\title{
Analytical Evaluation of a Vancomycin Immunoassay in Synovial Fluid
}

\author{
Francesca Luceri ${ }^{1} \quad$ Fiamma Balboni $^{2}$ Giovanni Balato ${ }^{3}$ Paola Pezzati $^{1} \quad$ Nicoletta Cini $^{1}$ Gianni Virgili ${ }^{4}$ \\ Andrea Baldini ${ }^{3}$
}

${ }^{1}$ Laboratorio Generale, Dipartimento dei Servizi, Azienda Ospedaliero Universitaria Careggi, Firenze, Italy

${ }^{2}$ Laboratorio Analisi, Istituto Fiorentino Cura E Assistenza, Firenze, Italy

3 UO Ortopedia, Istituto Fiorentino Cura E Assistenza, Firenze, Italy

${ }^{4}$ Dipartimento di Chirurgia e Medicina Translazionale, Università di

Firenze, Oculistica, Italy

Joints 2017;5:125-126.

\author{
Address for correspondence Paola Pezzati, MD, Laboratorio \\ Generale, Dipartimento dei Servizi, Azienda Ospedaliero Universitaria \\ Careggi, Firenze, Italy (e-mail: p.pezzati@live.it).
}

\begin{abstract}
Keywords

- vancomycin

- clinical laboratory services

- immunoassay synovial fluid

- antibacterial agents

In clinical laboratories performing routine activities, the need to answer the burning clinical question in emerging field may be limited by lack of technology support or assays accessibility. Commercially available methods, although originally validated for specific biological matrices, may be employed for other matrices, following appropriate guidelines such as Clinical and Laboratory Standards Institute (CLSI) EP 19. We investigated the use of a vancomycin assay with synovial fluid samples, in view of a possible employment in vancomycin release study. The standard of care of periprosthetic joint infection is a twostage revision surgery with antibiotic-loaded bone cement implantation. Vancomycin, for its activity against gram-positive bacteria even multidrug-resistant staphylococci, is the most widely used antibiotic. Despite the widespread use of such devices, little is known about the in vivo elution in the joint space. Clinical laboratories equipped with a validated, affordable method to quantify vancomycin in synovial fluid, may support clinical research, and give an important contribution to the study of the pharmacokinetics of antibiotic release from bone cement matrix.
\end{abstract}

\section{Introduction}

Clinical laboratories are required to answer several emerging clinical questions; however, any request for implementation, in terms of a new biomarker, innovative assay, or unconventional samples, and alternative matrices, needs to be evaluated according to best practice and to certification rules.

When instruments or reagents, intended for a specific sample matrix, are used to analyze a different matrix, laboratory personnel must validate the procedure, according to the international guideline such as Clinical and Laboratory Standards Institute (CLSI) EP19.

Clinical laboratories routinely perform serum vancomycin assay for therapeutic drug monitoring (TDM). Recently, assessment of vancomycin levels in synovial fluid from patient affected by periprosthetic joint infection (PJI) and undergoing revision surgery has been suggested. ${ }^{1}$
PJI is a rare yet devastating complication after total joint replacement, ranging between 0.5 and $3 \%$, with total knee arthroplasty (TKA) having a higher prevalence than total hip arthroplasty. ${ }^{2}$ Two-stage revision surgery with antibioticloaded spacer implantation is now considered the standard of care. ${ }^{3-5}$ Vancomycin is widely used and, although the pharmacokinetic properties of aminoglycosides have been thoroughly investigated in vitro and in vivo studies, ${ }^{5-8}$ only limited data are available on the vancomycin release in vivo from prosthetic devices after implantation. ${ }^{1}$ Accordingly, it appears to be of paramount importance the availability of an affordable and reliable analytical method to study vancomycin in vivo.

The aim of this study was to verify if vancomycin assay validated for TDM in human plasma and serum can be used to measure vancomycin in drainage fluid.
DOI https://doi.org/ 10.1055/s-0037-1603671. ISSN 2282-4324.
Copyright @ 2017 Georg Thieme Verlag KG Stuttgart · New York
License terms

()(1) $\Theta \circledast$ 


\section{Methods}

$\mathrm{K}_{2}$ EDTA blank drainage fluid samples were obtained from patients $(n=3)$ who underwent primary TKA. No systemic vancomycin was given before or after surgery in any patient. The samples collected 24 hours after surgery were pooled, centrifuged, transferred to Microcon YM10 filters (Merck Millipore, Billerica, Massachusetts, United States), designed to remove proteins from viscous biological samples. According to manufacturer instructions, filters were loaded and centrifuged at $15,000 \mathrm{~g}$ for 20 minutes, and the ultrafiltrates were then collected (Filtered Synovial Fluid [FSF]).

Vancomycin hydrochloride for injection USP $500 \mathrm{mg}$ was purchased from Mylan N.V. Pharmaceutical (Amsterdam, The Netherlands) and a stock solution of vancomycin $(1 \mathrm{mg} / \mathrm{mL})$ was prepared in blank FSF. Calibration standards at vancomycin concentrations of $5,10,25,50$, and $100 \mu \mathrm{g} / \mathrm{mL}$ were obtained from appropriate additions of stock solution to blank FSF.

Three levels of quality control $(2.5,25.0$, and $50.0 \mu \mathrm{g} / \mathrm{mL})$ were analyzed during each routine analysis, and these were prepared by the appropriate addition of another (independently prepared and weighed) stock solution of vancomycin $(1 \mathrm{mg} / \mathrm{mL})$.

All samples were analyzed on CDx90 (Thermo Scientific, Whaltam, Massachusetts, United States) instrument by Quantitative Microsphere System (QMS) vancomycin assay (Thermo Scientific); the vancomycin assay is a homogeneous particle-enhanced turbidimetric immunoassay, and it is based on competition between drug in the sample and drug coated onto a microparticle for antibody-binding sites of the vancomycin antibody reagent.

The vancomycin-coated microparticle reagent is rapidly agglutinated in the presence of the antivancomycin antibody reagent and the absence of any competing drug in the sample. The rate of absorbance change is measured photometrically.

When a sample containing vancomycin is added, the agglutination reaction is partially inhibited, slowing down the rate of absorbance change. A concentration-dependent classic agglutination inhibition curve can be obtained with a maximum rate of agglutination at the lowest vancomycin concentration and the lowest agglutination rate at the highest vancomycin concentration.

\section{Results}

The method was linear from 0 to $100 \mu \mathrm{g} / \mathrm{mL}\left(\mathrm{r}_{2}=0.987\right)$, selectivity was checked by analyzing 25 blank drainage fluid samples, the limit of quantification was $2.5 \mu \mathrm{g} / \mathrm{mL}$ and the accuracy ranged from 82 to $93 \%$ at three concentration levels $(2.5,25$, and $50 \mu \mathrm{g} / \mathrm{mL})$.

The precision of the method was determined by calculating the coefficient of variability at three concentration levels $(2.5,25$, and $50 \mu \mathrm{g} / \mathrm{mL})$ for 10 times in the same day (intraassay precision) and once for 10 days (inter-assay precision). Intra-assay precision ranged from 1.8 to $4.9 \%$ at each level and the inter-assay precision from 4.0 to $10.4 \%$.

\section{Discussion}

Since the introduction of antibiotic-loaded acrylic bone cement, antibiotic-impregnated bone cement have been widely used as a delivery vehicle for the local administration of antibiotics in joint-infected sites, reaching higher concentrations compared with systemic administration. ${ }^{5}$

The most commonly used antibiotics include tobramycin, gentamicin, vancomycin, and cephalosporins that show a wide spectrum activity even against the multidrug-resistant bacteria. Strikingly, a recent systematic review of the use of antibacterial cement spacers reports that a significantly high infection eradication rate, ranging from 73 to $100 \%$, can be achieved even when the antibacterials placed into the spacers are not active against the infecting organisms. ${ }^{5}$

Despite these appealing results, several questions still remain to be addressed to optimize the use of these devices and to define the ideal moment for prosthetic reimplantation. Indeed, the therapeutic concentration levels of antibiotics present at the implantation site and the actual inhibitory activity of antibiotics released at the infection site, in the first postoperative period, are still open questions, along with the duration of the antimicrobial effect.

The possibility to measure vancomycin levels in drainage fluid is the basis for studying the antibiotic release in vivo from prosthetic devices after implantation and might represent a step ahead toward treatment optimization. Nevertheless, significant heterogeneity caused by differences in methods and experimental conditions have complicated the attempts to draw definite conclusions from available studies. The QMS method guarantees enough precision and accuracy at low costs to answer, at least, some of the clinical questions related to PJIs.

\section{References}

1 Bertazzoni Minelli E, Benini A, Samaila E, Bondi M, Magnan B. Antimicrobial activity of gentamicin and vancomycin combination in joint fluids after antibiotic-loaded cement spacer implantation in two-stage revision surgery. J Chemother 2015;27(01):17-24

2 Del Pozo JL, Patel R. Clinical practice. Infection associated with prosthetic joints. N Engl J Med 2009;361(08):787-794

3 Leone JM, Hanssen AD. Management of infection at the site of a total knee arthroplasty. J Bone Joint Surg Am 2005;87(10):2335-2348

4 Cui Q, Mihalko WM, Shields JS, Ries M, Saleh KJ. Antibioticimpregnated cement spacers for the treatment of infection associated with total hip or knee arthroplasty. J Bone Joint Surg Am 2007;89(04):871-882

5 Iarikov D, Demian H, Rubin D, Alexander J, Nambiar S. Choice and doses of antibacterial agents for cement spacers in treatment of prosthetic joint infections: review of published studies. Clin Infect Dis 2012;55(11):1474-1480

6 Balato G, Ascione T, Rosa D, et al. Release of gentamycin from cement spacers in two-stages procedure for hip and knee prosthetic infection: an in vivo pharmacokinetic study with clinical follow-up.J Biol Regul Homeost Agents 2015;29(4, Suppl):63-72

7 Anagnostakos K, Kelm J. Enhancement of antibiotic elution from acrylic bone cement. J Biomed Mater Res B Appl Biomater 2009; 90(01):467-475

8 Sterling GJ, Crawford S, Potter JH, Koerbin G, Crawford R. The pharmacokinetics of Simplex-tobramycin bone cement. J Bone Joint Surg Br 2003;85(05):646-649 